\title{
Homeobox gene TGIF-1 is increased in placental endothelial cells of human fetal growth restriction
}

\author{
Tilini Gunatillake ${ }^{1,2, *}$, Hannah E J Yong ${ }^{1,2, *}$, Caroline E Dunk ${ }^{3}$, Rosemary J Keogh ${ }^{1,2}$, \\ Anthony J Borg ${ }^{1}$, Judith E Cartwright ${ }^{4}$, Guy S Whitley ${ }^{4}$ and Padma Murthi ${ }^{1,2,5,6}$ \\ ${ }^{1}$ Department of Maternal-Fetal Medicine Pregnancy Research Centre, The Royal Women's Hospital, Parkville, \\ Victoria, Australia, ${ }^{2}$ Department of Obstetrics and Gynaecology, The University of Melbourne, Parkville, Victoria, \\ Australia, ${ }^{3}$ Lunenfeld Tanenbaum-Research Institute, Mount Sinai Hospital, Toronto, Ontario, Canada, ${ }^{4}$ Institute of \\ Cardiovascular and Cell Sciences, St George's, University of London, London, UK, ${ }^{5}$ Department of Medicine, \\ School of Clinical Sciences, Monash University, Clayton, Victoria, Australia and ${ }^{6}$ The Ritchie Centre, Hudson \\ Institute of Medical Research, Clayton, Victoria, Australia
}

Correspondence should be addressed to P Murthi; Email: padma.murthi@monash.edu

*(T Gunatillake and H E J Yong contributed equally to this work)

\begin{abstract}
Aberrant placental angiogenesis is associated with fetal growth restriction (FGR). In mice, targeted disruption of the homeobox gene, transforming growth $\beta$-induced factor (Tgif-1), which is also a transcription factor, causes defective placental vascularisation. Nevertheless, the role of TGIF-1 in human placental angiogenesis is unclear. We have previously reported increased TGIF-1 expression in human FGR placentae and demonstrated localisation of TGIF-1 protein in placental endothelial cells (ECs). However, its functional role remains to be investigated. In this study, we aimed to specifically compare TGIF-1 mRNA expression in placental ECs isolated from human FGR-affected pregnancies with gestation-matched control pregnancies in two independent cohorts from Australia and Canada and to identify the functional role of TGIF-1 in placental angiogenesis using the human umbilical vein endothelial cell-derived cell line, SGHEC-7, and primary human umbilical vein ECs. Real-time PCR revealed that TGIF-1 mRNA expression was significantly increased in ECs isolated from FGR-affected placentae compared with that of controls. The functional roles of TGIF-1 were determined in ECs after TGIF-1 siRNA transfection. TGIF-1 inactivation in ECs significantly reduced TGIF-1 at both the mRNA and protein levels, as well as the proliferative and invasive potential, but significantly increased the angiogenic potential. Using angiogenesis PCR screening arrays, we identified ITGAV, NRP-1, ANPGT-1 and ANPGT-2 as novel downstream targets of TGIF-1, after TGIF-1 inactivation in ECs. Collectively, these results show that TGIF-1 regulates EC function and the expression of angiogenic molecules; and when abnormally expressed, may contribute to the aberrant placental angiogenesis observed in FGR. Reproduction (2016) 152 457-465
\end{abstract}

\section{Introduction}

Placental angiogenesis is critical for maintaining the highly efficient transport system that facilitates the exchange of nutrients, oxygen and waste between the mother and the fetus (Reynolds \& Redmer 2001). Endothelial cells (ECs) play significant roles in coordinating effective angiogenesis, and aberrant EC function can lead to pregnancy pathologies such as fetal growth restriction (Kingdom et al. 2000, Bouis et al. 2001). Impaired placental angiogenesis is observed in FGR, with reduced tubule length and formation in placental ECs obtained from FGR-affected pregnancies (Su et al. 2015). Placental angiogenesis is tightly controlled by numerous growth factors, cytokines and signalling pathways that collectively regulate expression of multiple genes through the activation of transcription factors (Latchman 1997, Hamik et al. 2006).
Transcription factors that regulate angiogenesis include the family of homeobox genes (Gorski \& Walsh 2000). Homeobox genes belong to a highly conserved family of transcription factors (Holland et al. 2007), which control cell and organ differentiation throughout embryonic development (Yaron et al. 2001) and have pleiotropic effects on cell proliferation, growth arrest and differentiation (Gorski \& Walsh 2000, Douville \& Wigle 2007). Mouse knockout studies demonstrate the involvement of homeobox genes in regulating placental functions (Rossant \& Cross 2001). Previous studies in our laboratory have identified that several homeobox genes including DLX3, HOXB6, DLX4, MSX2, GAX and HLX1 are expressed in ECs surrounding the fetal capillaries in the human placenta (Murthi et al. 2007). We have also demonstrated the expression of several novel homeobox genes in placental ECs including the novel transforming 
growth $\beta$-induced factor (TGIF-1) homeobox gene at the mRNA level (Murthi et al. 2008).

TGIF-1 is a negative regulator of the transforming growth factor beta (TGF- $\beta$ ) pathway (Faresse et al. 2008), which is important for physiological processes such as cell proliferation, differentiation, apoptosis, early development and placental angiogenesis. Missense mutations in the TGIF-1 gene can lead to holoprosencephaly, which affects cranial development (Hayhurst \& McConnell 2003). In addition, mouse studies show that Tgif-1-null embryos display a severely growth-restricted phenotype associated with placental vascular defects (Bartholin et al. 2008). Our recent study showed a significantly increased TGIF-1 expression in FGR placental homogenates at both the mRNA and protein levels, with protein localisation of TGIF-1 in the endothelium lining the fetal capillaries (Pathirage et al. 2013). However, the role of TCIF-1 in human placental ECs is largely unknown.

In this study, we hypothesised that TGIF-1 is an important regulator of placental angiogenesis. A previous microarray analysis on placental endothelial cells (PLECs) from FGR pregnancies performed in Toronto, Canada, demonstrated a trend for increased TGIF-1 mRNA expression in the FGR PLEC samples compared with that of the controls (Dunk et al. 2012). In this study, using the two independent cohorts from Toronto, Canada and from samples collected in Melbourne, Australia, ECs isolated from FGR placentae and control placentae were further investigated and validated for TGIF-1 expression. We aimed to determine the level of TGIF- 1 mRNA expression in placental ECs isolated from FGR and gestationmatched control (GMC) pregnancies in two independent cohorts and to investigate the functional role of TGIF-1 by gene inactivation in primary human umbilical vein endothelial cells (HUVECs) and the HUVEC-derived cell line, SGHEC-7 (Fickling et al. 1992).

\section{Materials and methods}

\section{Placental endothelial cell (PLEC) isolation}

PLECs were isolated and purified from two independent cohorts of placentae from Melbourne, Australia and Toronto, Canada as described previously (Dunk et al. 2012). Placentae were collected from FGR $(n=10)$ and GMC $(n=10)$ patients in the Australian study arm and FGR $(n=6)$ and GMC $(n=4)$ patients in the Canadian study arm after obtaining written informed consent. Patient characteristics of the Australian samples are presented in Table 1. Using these placental samples, previous studies in our laboratory have shown consistent gene expression changes in the placental villi of FGR compared with those of gestation-matched control pregnancies (Murthi et al. 2006, Pathirage et al. 2013). FGR was defined as birth weight less than the 10th centile for gestational age according to Australian growth charts (Murthi et al. 2006) accompanied by two or more of the following features: abnormal umbilical artery Doppler flow velocimetry,
Table 1 Clinical characteristics of Australian samples

\begin{tabular}{lccc}
\hline Characteristics & GMC $(n=10)$ & FGR $(n=10)$ & $\boldsymbol{P}_{\text {value }}^{\dagger}$ \\
\hline Maternal age (years) & $34.8 \pm 1.6$ & $32.7 \pm 2.0$ & 0.41 \\
Gestation at delivery & $38.7 \pm 0.3$ & $38.4 \pm 0.4$ & 0.52 \\
$\quad$ (weeks) & & & \\
Infant weight (g) & $3406.3 \pm 122.8$ & $2448.7 \pm 63.3$ & $<0.0001$ \\
Placental weight (g) & $662.4 \pm 32.8$ & $471.3 \pm 28.8$ & $<0.001$ \\
Parity & 5 primi, 5 multi & 4 primi, 6 multi & 1.00 \\
Infant sex & 6F, 4M & 9F, 1M & 0.30 \\
\hline
\end{tabular}

Data is presented as mean \pm S.E.M. with ranges shown in brackets unless stated otherwise; ${ }^{\top}$ Student's $t$-test for parametric data and $2 \times 2$ contingency table with Fisher's exact test for categorical data were used where appropriate.

oligohydramnios as determined by amniotic fluid index (AFI) of $<7$ and asymmetric growth of the fetus as defined by a head circumference (HC)-abdominal circumference (AC) ratio $>1.2$. The exclusion criteria for both FGR and GMC pregnancies were multiple pregnancies, illicit drug dependency, maternal smoking, pre-eclampsia, prolonged rupture of the membranes, placental abruption, intrauterine viral infection and fetal congenital anomalies. The Australian samples were collected with approval from the Royal Women's Hospital Human Research Ethics Committee (Project \# 27/00) in Melbourne, Australia. All Canadian samples were collected by the Research Centre for Women's and Infants' Health BioBank Program (http://biobank.lunenfeld.ca/) with the approval of the Research Ethics Board at Mount Sinai Hospital (04-0018-U) in Toronto, Canada, and the FGR and matched control samples were previously characterised by Dunk and coworkers (2012). All FGR cases in the Canadian cohort were presented with abnormal umbilical Doppler velocimetry. Freshly isolated cells were used for gene expression analyses.

\section{HUVEC isolation}

Human umbilical vein endothelial cells (HUVECs) were freshly isolated from uncomplicated term pregnancies $(n=12)$ as described previously (Murthi et al. 2008). Briefly, cells were cultured and maintained in M199 tissue culture medium (Thermo Fisher Scientific) with $10 \%$ fetal bovine serum supplemented with $2 \mathrm{mM}$ L-glutamine, $100 \mathrm{U} / \mathrm{mL}$ penicillin and $100 \mu \mathrm{g} / \mathrm{mL}$ streptomycin and maintained in $5 \% \mathrm{CO}_{2} / 95 \%$ air. For gene expression and functional analyses, HUVEC cells from passage 2 were used.

\section{SYBR green qPCR analysis on Canadian cohort}

SYBR green q-PCR was performed as described previously (Dunk et al. 2012). Briefly, $1 \mu$ g total RNA from isolated PLECs was reverse transcribed using the iScript cDNA synthesis kit (Bio-Rad Laboratories). Approximately $10 \mathrm{ng}$ of each cDNA were then subjected to real-time PCR using primers specific for TGIF (forward 5'-TCGGTGTGGGACAAAACACA-3' and reverse 5'-TCGGTGTGGGACAAAACACA-3') and housekeeping genes SDHA and YWHAZ (Dunk et al. 2012). Real-time PCR was performed in a white 96-well plate in a CFX96 real-time PCR system (Bio-Rad Laboratories). The run protocol was as follows: heat activation of Taq and denaturation at $95^{\circ} \mathrm{C}$ for $2 \mathrm{~min}$ and 40 cycles of amplification at $95^{\circ} \mathrm{C}$ for 
$10 \mathrm{~s}$ and $60^{\circ} \mathrm{C}$ for $30 \mathrm{~s}$. The mRNA level of the gene of interest from each sample was normalised to the geometric mean of the YWHAZ and SDHA mRNA expression level, and data were analysed using the $2^{-\triangle \Delta C T}$ method (Livak \& Schmittgen 2001).

\section{RNA extraction, cDNA synthesis, real-time PCR of Australian cohort and cell lysates}

Total cellular RNA was extracted from the Australian cohort of PLECs and EC lysates using the PureLink RNA Mini Kit (Thermo Fisher Scientific) following the manufacturer's instructions. RNA yield, purity and integrity were determined by visualising $28 \mathrm{~S}$ and $18 \mathrm{~S}$ ribosomal RNA following $1 \%(\mathrm{w} / \mathrm{v})$ agarose gel electrophoresis. First-strand cDNA was prepared as described previously (Rajaraman et al. 2010). Real-time PCR was performed using FAM-labelled TaqMan probes (ANGPT-1 Hs00375823_m1; ANGPT-2 Hs00169867_m1; ITGAV Hs00233808_m1; NRP-1 Hs00826129_m1 and TGIF1 Hs00545233_m1) and Eukaryotic 18S rRNA Endogenous Control (VIC/MGB Probe, Primer Limited) as a housekeeping gene in an $\mathrm{ABI}$ PRISM 7500HT thermocycler (Applied Biosystems). Approximately $12.5 \mathrm{ng} / \mu \mathrm{L}$ cDNA were amplified in a total reaction volume of $20 \mu \mathrm{L}$. PCR conditions included an activation cycle of $50^{\circ} \mathrm{C}$ for $2 \mathrm{~min}$ and $95^{\circ} \mathrm{C}$ for $10 \mathrm{~min}$ followed by 40 cycles of $95^{\circ} \mathrm{C}$ for $15 \mathrm{~s}$ and $60^{\circ} \mathrm{C}$ for $1 \mathrm{~min}$. Relative mRNA expression for each gene to the $18 \mathrm{~S}$ rRNA housekeeping gene was determined using the $2^{-\Delta \Delta C T}$ method (Livak \& Schmittgen 2001).

\section{Cell culture}

The well-characterised HUVEC-derived cell line, SGHEC-7, was cultured as described previously (Fickling et al. 1992). Briefly, cells were grown in a 1:1 mixture of RPMI-1640 and medium 199 supplemented with 10\% (v/v) heat-inactivated fetal bovine serum, $1 \%(\mathrm{w} / \mathrm{v})$ L-glutamine, $100 \mathrm{lU} / \mathrm{mL}$ penicillin and $100 \mathrm{mg} / \mathrm{mL}$ streptomycin. Cells were maintained in controlled, humidified conditions at $37^{\circ} \mathrm{C}$ in $5 \% \mathrm{CO}_{2}$ and 95\% air.

\section{Immunocytochemistry}

Immunocytochemistry was performed using the Zymed Histostain-plus Broad Spectrum kit (Thermo Fisher Scientific) as described previously (Lepparanta et al. 2010). Mouse antiTGIF-1 monoclonal IgG (0.02 $\mu \mathrm{g} / \mu \mathrm{L}$; Santa Cruz Biotechnology) in $2 \%(\mathrm{w} / \mathrm{v})$ non-fat milk in phosphate buffered saline was used to detect TGIF-1 protein. Mouse IgG2b (Dako) was used as a negative control. Colour detection was performed using the Zymed AEC chromogen kit (Thermo Fisher Scientific), and slides were mounted with $80 \%(\mathrm{v} / \mathrm{v})$ glycerol. Cells were viewed with a Zeiss Axioscope microscope, and images were captured with a Zeiss Axiocam camera and analysed using Axiovision Rel. 4.3 software (Carl Zeiss AG).

\section{TGIF-1 inactivation in ECs}

Two independently validated siRNAs, TGIF-1 siRNA-1 (S1) and TGIF-1 siRNA-2 (S2) from Life Technologies, were used to silence TGIF- 1 expression in ECs. TGIF- 1 oligonucleotides were diluted to $80 \mu \mathrm{M}$ with RNAiFect transfection reagent (Qiagen) added dropwise to cells grown in 6-well plates and incubated for $72 \mathrm{~h}$ in culture. AllStars Negative Control siRNA (Qiagen) that had no homology to any known mammalian gene was used as a negative control (NC).

\section{Protein extraction and Western immunoblotting}

Whole-cell protein was extracted using radioimmunoassay precipitation assay buffer containing $50 \mathrm{mM}$ Tris- $\mathrm{HCl}, 150 \mathrm{mM}$ $\mathrm{NaCl}, 1 \%(\mathrm{v} / \mathrm{v})$ Triton-X-100, 1\% (w/v) sodium deoxycholate and $0.1 \%(\mathrm{w} / \mathrm{v})$ sodium dodecyl sulphate supplemented with $1 \mathrm{X}$ protease and $1 \mathrm{X}$ phosphatase inhibitor cocktails (Roche). Immunoblotting was then performed as described previously (Murthi et al. 2006). Mouse anti-TGIF-1 monoclonal IgG $(0.02 \mu \mathrm{g} / \mu \mathrm{L}$; Santa Cruz Biotechnology) and rabbit anti-GAPDH polyclonal IgG (1.25 ng/ $\mathrm{LL}$; Imgenex Corp, San Diego, CA, USA) were used to detect TGIF-1 and GAPDH protein, respectively. Horseradish peroxidase (HRP)-conjugated goat anti-mouse IgGs ( $1 \mathrm{ng} / \mu \mathrm{L}$; Thermo Fisher Scientific) or HRP-conjugated goat anti-rabbit IgGs $(1.5 \mathrm{ng} / \mu \mathrm{L}$, Thermo Fisher Scientific) were used as secondary antibodies. Immunoreactivity was detected using an enhanced chemiluminescence system (GE Healthcare) and the luminescence detector LAS-4000 image reader (Fujifilm Corp, Tokyo, Japan). Immunoreactive protein bands were quantitated using the Image software. Levels of TGIF-1 was normalised to that of GAPDH to control for protein loading.

\section{Cell proliferation assay}

Cell proliferation was assessed using the CellTiter 96 Aqueous One Cell Proliferation Assay (Promega) following the manufacturer's instructions. Briefly, $2 \times 10^{4}$ SGHEC-7 cells were transfected with either TGIF-1 siRNA-1 or SiRNA-2 and plated in a 96-well plate in serum-free medium. After $24 \mathrm{~h}$ of culture (72 $\mathrm{h}$ after transfection), the cell proliferation assay was performed and the plate read at $\lambda_{490}$.

\section{Zymography}

The effect of TGIF-1 siRNA inactivation on the activity of metalloproteinases was assessed using gelatin-based zymography as described previously (Fitzsimmons et al. 2007). Dehydrated gels were scanned on an ImageScanner III densitometer (GE Healthcare), and band intensities were quantified using the ImageQuant software provided with the instrument.

\section{Network formation assay}

To observe the effect of TGIF-1 inactivation on the network formation ability of ECs, 24-well plates were coated with a thin layer of undiluted growth factor-reduced Matrigel (BD Biosciences, San Jose, CA, USA). At $48 \mathrm{~h}$ after transfection, $2 \times 10^{5}$ cells were seeded onto the pre-coated plates and incubated in complete basal medium for further $24 \mathrm{~h}$ as described previously (Arnaoutova \& Kleinman 2010). Images 
were taken every $4 \mathrm{~h}$ throughout the $24 \mathrm{~h}$ using the Incucyte Live Cell Imaging System (Essen Bioscience, Ann Arbor, MI, USA) at a magnification of $200 \times$.

\section{Angiogenesis array}

The effect of TGIF-1 silencing on angiogenic genes was determined using TaqMan human angiogenesis signature arrays (Applied Biosystems), which consisted of 92 human genes involved in the regulation of angiogenesis. Gene profiling was used to identify the downstream target genes of TGIF-1, and the methodology was carried out according to the manufacturer's instructions. Briefly, cDNA was prepared using an $\mathrm{RT}^{2}$ FirstStrand kit and added to a TaqMan Universal Master Mix, which contained the AmpliTaq Gold DNA polymerase and optimised buffer components (Applied Biosystems). Housekeeping genes consisted of $\beta$-2-microglobulin (B2M), $18 S$ rRNA, glyceraldehyde-3-phosphate dehydrogenase $(G A P D H)$ and $\beta$-actin $(A C T B)$. The quantification of relative gene expressions was performed on an $\mathrm{ABI}$ Prism 7700 Sequence Detector (Applied Biosystems) under the cycling parameters: $95^{\circ} \mathrm{C}$ for $10 \mathrm{~min}$, followed by 40 cycles of denaturation at $95^{\circ} \mathrm{C}$ for $15 \mathrm{~s}$ and primer extension at $60^{\circ} \mathrm{C}$ for $1 \mathrm{~min}$. Data $\left(\mathrm{C}_{\mathrm{t}}\right.$ values $)$ were analysed using the $\mathrm{ABI}$ Sequence Detector System software version 2.0 (Applied Biosystems) and the relative gene expression values, or fold changes, were calculated according to the manufacturer's protocols. Briefly, values from the SGHEC-7 control (NC siRNA) and treated plates (TCIF-1siRNA) were calculated as $2^{-\Delta \Delta \mathrm{Ct}}$ (Livak \& Schmittgen 2001), and normalised to the average $C_{t}$ value of the housekeeping genes. Target genes of TGIF-1 were identified by calculating the fold change in gene expression levels for TGIF-1 siRNAtreated cells relative to the NC. Candidate genes that showed either a fold-change increase of $>2$ or a decrease of $<2$ in gene expression were identified. Data were analysed and compared with the NC transfected SGHEC-7 cells using Data Assist (Applied Biosystems).

\section{Statistical analysis}

Data are shown as mean \pm S.E.M. of $n \geq 3$ independent experiments. Student's $t$-tests, $2 \times 2$ contingency table with Fisher's Exact Test and ANOVA with Bonferroni's post-test were carried out using GraphPad Prism 5 (GraphPad Software). A value of $P<0.05$ was considered to be statistically significant.

\section{Results}

\section{Clinical characteristics}

Table 1 describes the clinical characteristics of FGR $(n=10)$ and GMC $(n=10)$ patients, whose placentae were collected and used in the Australian arm of this study. As shown, there were no significant differences in the gestational age, maternal age or infant sex between FGRs and controls. FGR cases showed significantly lower birth weights and lower placental weights. In addition, all FGR subjects had a birth weight below the 10th percentile for gestational age (Dobbins et al.
2012) and either abnormal umbilical artery Doppler findings or oligohydramnios together with evidence of asymmetric growth (head circumference: abdominal circumference $>95$ th centile). The Canadian FGR samples were associated with abnormal umbilical artery Doppler changes in all $n=6$ cases as described previously by Dunk and coworkers (2012).

\section{Increased TGIF-1 expression in FGR placentae}

Real-time PCR was used to validate the initial microarray data, which showed a trend towards increased expression in TGIF-1 mRNA. TGIF-1 mRNA expression was determined in two independent cohorts of FGR and control PLECs from Melbourne, Australia and Toronto, Canada. As shown in Fig. 1, significant increases in TGIF-1 mRNA of 2.4-fold and 1.5-fold were observed in PLECs isolated from FGR pregnancies compared with those from control pregnancies in the Australian (Fig. 1A) and Canadian (Fig. 1B) cohorts respectively.
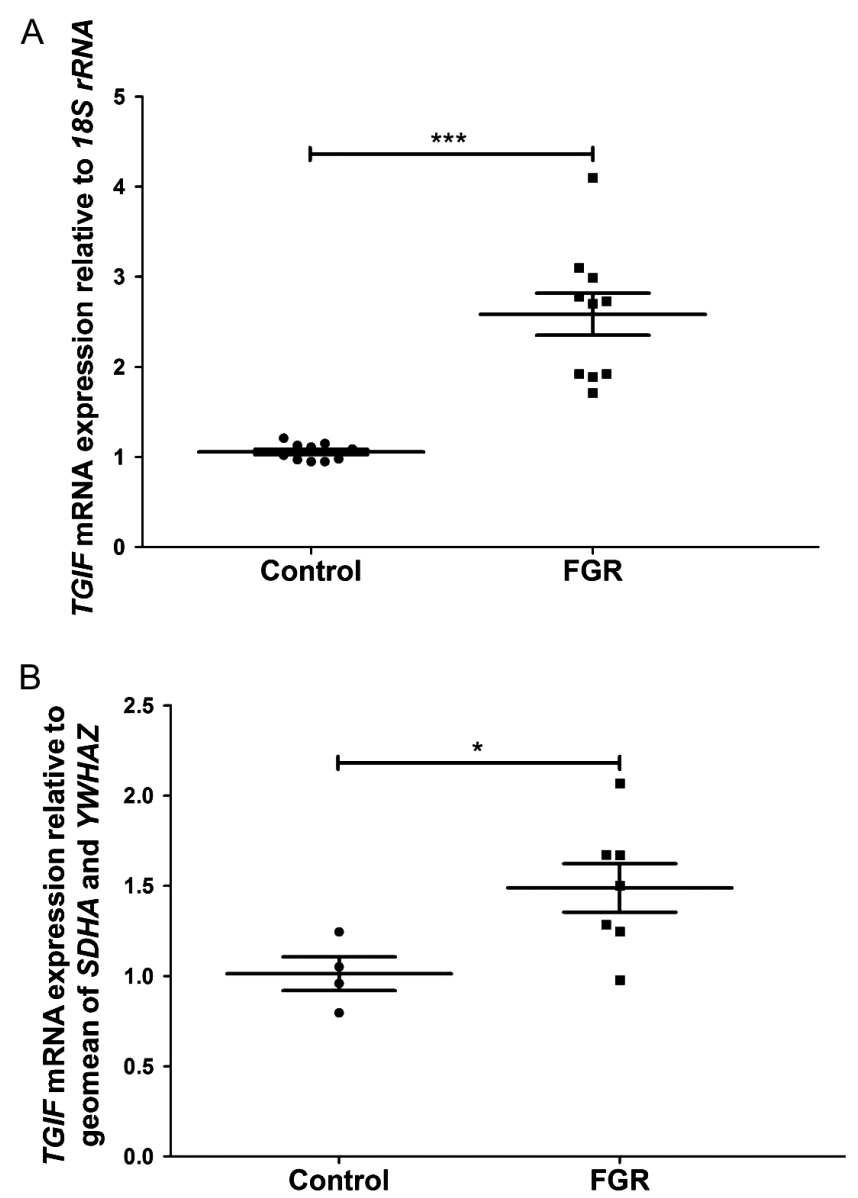

Figure 1 Increased TGIF-1 mRNA expression relative to housekeeping genes in endothelial cells from FGR compared with control placentae was determined by real-time PCR analysis in two independent cohorts from Melbourne, Australia (A) and Toronto, Canada (B). ${ }^{*} P<0.05,{ }^{* * *} P<0.001$, Student's $t$-test. 


\section{TGIF-1 is expressed in HUVEC and in the SGHEC-7 cell line}

Immunocytochemistry was used to demonstrate the expression and localisation of TGIF-1 protein in HUVECs and SGHEC-7 cells, which were used as the in vitro cell culture model. As apparent in Fig. 2A (TGIF), TGIF-1 protein localised to the cell nuclei. Substitution of the primary antibody with mouse $\lg \mathrm{G} 2 \mathrm{~b}$ control showed no such immunoreactivity (Fig. 2A, IgG control).

\section{TGIF-1 silencing in HUVEC and in the SGHEC-7 cell line}

After transient siRNA transfection of HUVECs and SGHEC-7 cells for $72 \mathrm{~h}$, both TGIF-1-specific siRNAs (denoted as S1 and S2) significantly decreased TGIF-1 mRNA expression in comparison with the NC-transfected cells (Fig. 2B and C). This decrease in mRNA expression was further confirmed by examining the TGIF-1 protein expression in HUVECs SGHEC-7 cells after transfection. As shown in Fig. 2D, a decrease in immunoreactive TGIF-1 protein at $35 \mathrm{kDa}$ was evident in TGIF- 1 siRNAtreated cells compared with that of NC-treated cells, with no change in GAPDH housekeeping protein loading control. Densitometric analysis confirmed a significant decrease in the protein expression of TGIF-1 in both $\mathrm{S} 1$ and S2 compared with NC-treated cells (Fig. 2E and F).

\section{TGIF-1 silencing decreases endothelial cell proliferation}

After the siRNA inactivation of TGIF- 1 in HUVECs and SGHEC-7cells, serum-starved cells were assessed for their proliferative ability over $24 \mathrm{~h}$. As shown in Fig. 3A and B, TGIF-1 inactivation significantly decreased the proliferative potential of these cells compared with that of the NC.

\section{TGIF-1 silencing increases network formation}

The effect of TGIF-1 inactivation on the angiogenic potential of HUVECs and SGHEC-7 cells was assessed by network formation assays. Angiogenic potential was determined by assessing branch points at the 4-h time point. Significantly increased angiogenic potential was observed in HUVECs and SGHEC-7 cells transfected with either S1 or S2 compared with that in NC-transfected cells (Fig. 3C, D and E).

\section{TGIF-1 silencing decreases SGHEC-7 invasive potential}

The matrix metalloproteinase activities of MMP-2 and MMP-9 in SGHEC-7 cells were determined by gelatin zymography and used as proxies for invasive potential. Activities of MMP-2 and -9 were assessed in the culture medium collected from TGIF-1-inactivated SGHEC-7 cells (S1 and S2) and compared with medium from NC-transfected cells (Fig. 3F, G and H). TCIF-1 inactivation significantly decreased MMP-2 activity (Fig. 3F) compared with the NC $(P<0.05$, ANOVA), but did not alter MMP-9 activity (Fig. 3G). This was further confirmed in primary HUVEC after TGIF-1 inactivation using S1 siRNA compared with NC-treated cells. MMP2 activity in S1-treated cells demonstrated a significant reduction (34\%) in MMP2 activity $(98.67 \pm 1.7$ (NC) vs $33.67 \pm 3.8(\mathrm{~S} 1), n=3, P<0.05)$ compared with $\mathrm{NC}$, whereas no significant difference in MMP9 activity was observed in S1-treated cells compared with that in NC-treated HUVEC cells $(100.0 \pm 2.5$ (NC) vs $87.67 \pm 4.9$ (S1) $n=3, P=0.09$ ).

\section{Downstream targets of TGIF-1 in endothelial cells}

The human angiogenesis array consisting of 92 human genes involved in the regulation of angiogenesis was used to identify the potential downstream targets of TGIF-1. After TGIF-1 inactivation with siRNA in SGHEC-7 cells, 51 genes were upregulated, whereas 19 genes were downregulated. Changes in gene expression of four prioritised genes (ITGAV, NRP-1, ANGPT-1 and $A N C P T-2)$ were then validated with real-time PCR in HUVECs and SGHEC-7 cells (Fig. 4). ITGAV mRNA and NRP-1 mRNA were significantly increased in
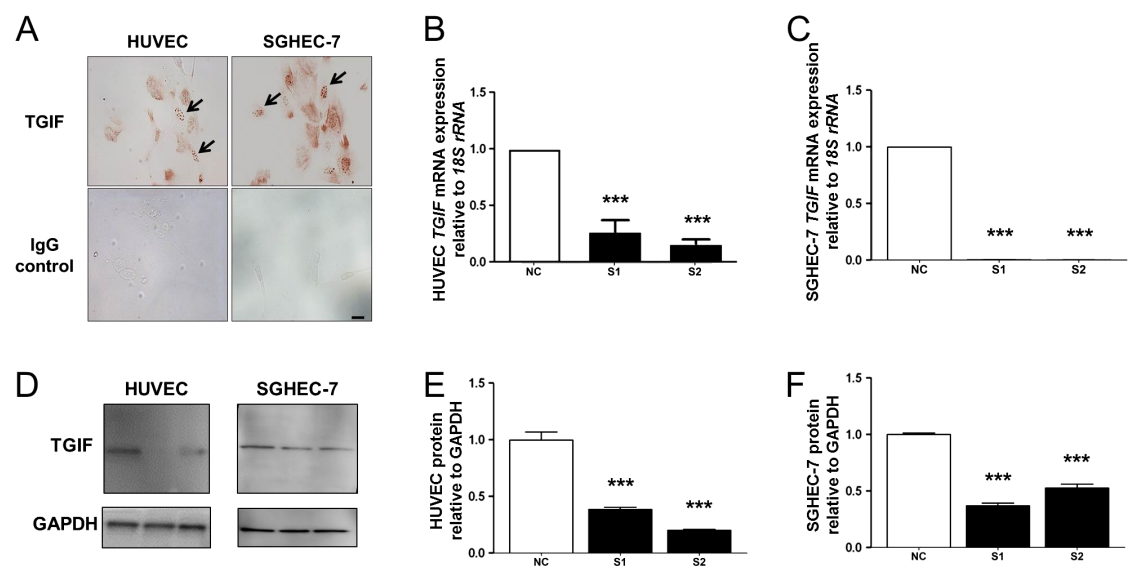

Figure 2 TGIF-1 expression in the primary HUVECs and the SGHEC-7 cell line. Immunoreactive TGIF-1 localised to the nuclei in HUVECs and SGHEC-7 cells as denoted by black arrows, which is absent in the negative $\lg G$ control (A). Scale bar represents $100 \mu \mathrm{m}$. siRNA inactivation which resulted in reduced TGIF-1 mRNA expression in HUVECs (B) and SGHEC-7 cells (C), which was validated using Western immunoblotting (D) and confirmed at the protein level ( $E$ and F). NC denotes the non-specific siRNA used as the negative control, whereas S1 and S2 refer to the two independent TGIF-specific siRNAs used in the siRNA transfection experiments. ${ }^{* * *} P<0.001$, one-way ANOVA with Bonferroni's post-test. 

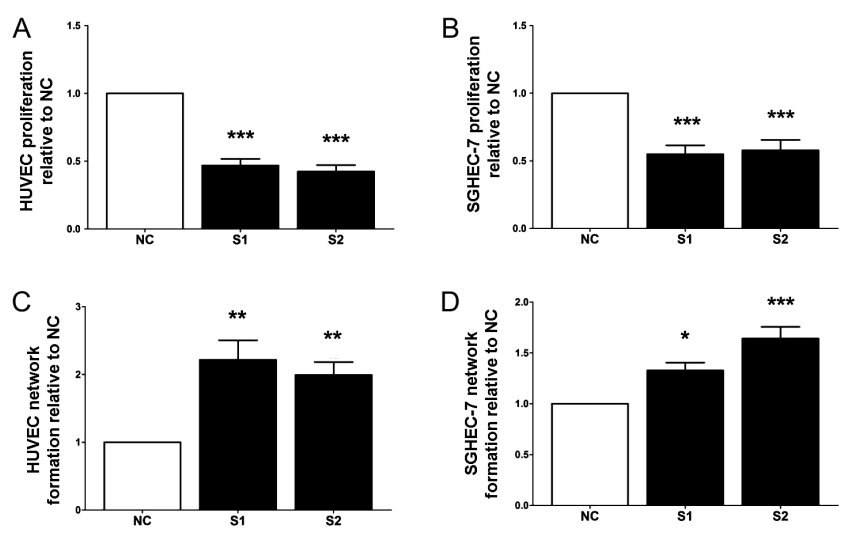

E
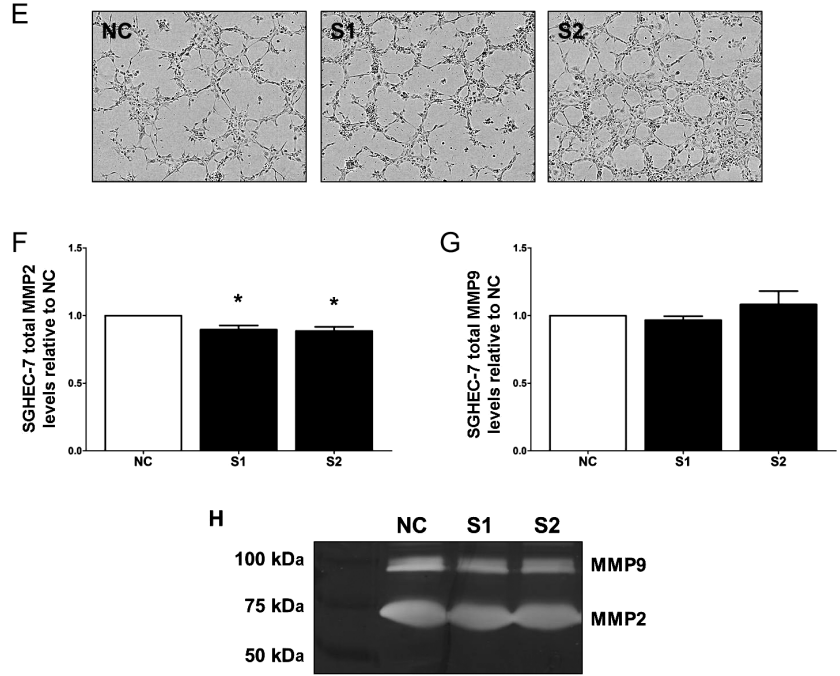

Figure 3 Functional analyses of TGIF-1 in HUVECs and SGHEC-7 cells. TGIF-1 siRNA inactivation significantly reduced proliferation ( $A$ and $B$ ), increased tube formation $(C, D$ and $E$ ) and decreased MMP-2 activity (F), with no effect on MMP-9 activity (G) of ECs. Representative experiments of the tube formation assay and zymography are presented in $\mathrm{E}$ and $\mathrm{H}$ respectively. $\mathrm{NC}$ denotes the non-specific siRNA used as the negative control, whereas S1 and S2 refer to the two independent TGIF-specific siRNAs used in the siRNA transfection experiments. ${ }^{*} P<0.05,{ }^{*} P<0.01,{ }^{* * *} P<0.001$, one-way ANOVA with Bonferroni's post-test.

TGIF-1 siRNA-transfected HUVECs (Fig. 4A and C), with a similar trend observed in the SGHEC-7 cells (Fig. 4B and D). Expression of ANGPT-1 mRNA was significantly decreased in both TGIF-1-inactivated HUVECs (Fig. 4E) and SGHEC-7 cells (Fig. 4F). In contrast to ANGPT-1, ANGPT-2 mRNA was significantly increased in TGIF-1inactivated HUVECs (Fig. 4G), although no significant change was observed in that of SGHEC-7 cells (Fig. 4H).

\section{Discussion}

Homeobox genes are important in the regulation of numerous vascular cell processes such as cell migration, invasion and proliferation (Douville \& Wigle 2007). Previous studies in our laboratory reported a range of novel placental homeobox genes expressed
A

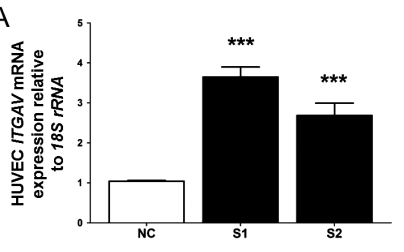

C
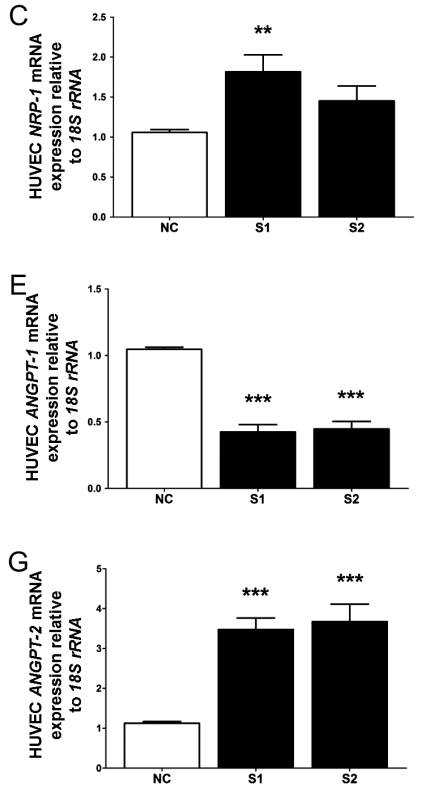

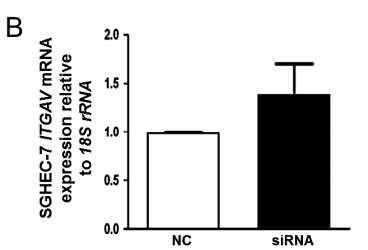

。
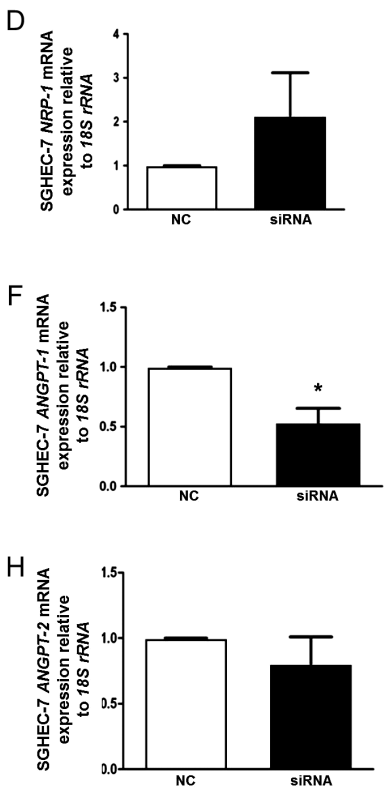

Figure 4 Validation of angiogenesis gene screen after TGIF-1 siRNA inactivation in HUVECs and the SGHEC-7 cell line. Altered mRNA expression of ITGAV (A and B), NRP-1 (C and D), ANGPT-1 (E and F) and ANGPT-2 (G and $\mathrm{H}$ ) were verified using real-time PCR after normalising to the $18 S$ rRNA housekeeping gene. NC denotes the non-specific siRNA used as the negative control, whereas S1 and S2 refer to the two independent TGIF-specific siRNAs used in the siRNA transfection experiments. ${ }^{*} P<0.05,{ }^{* *} P<0.01,{ }^{* * *} P<0.001$, Student's $t$-test.

in both microvascular and macrovascular ECs (Murthi et al. 2008). One such example is homeobox gene TGIF-1, which is expressed at the mRNA level in both macrovascular ECs and microvascular ECs. Studies from our laboratory demonstrated a significant increase in TGIF-1 in placentae from idiopathic FGR-affected pregnancies compared with that from uncomplicated control pregnancies. However, the functional role of TGIF- 1 in human placentae, in particular, its role in placental angiogenesis, is unclear. Therefore, the focus of this study was to investigate the functional role and the angiogenic downstream targets of TGIF-1.

Nuclear expression of TGIF-1 protein in the primary HUVECs and the SGHEC-7 cell line, which were used as an in vitro EC model, was confirmed using immunocytochemistry. Successful knockdown of TGIF-1 expression at both the mRNA and protein levels was achieved using two independent TGIF-1 siRNAs to inactivate the TGIF-1 gene expression in ECs. Previous studies have demonstrated the angiogenic potential of the homeobox gene HEX 
in HUVECs (Nakagawa et al. 2003). This study found overexpression of HEX disrupted the ability of the ECs to migrate, proliferate and form tubular structures in response to VEGF stimulation. Apart from this study, limited information exists in the literature about the effects of modulating expression of homeobox genes on EC function in either HUVEC or in human placental microvascular ECs. Therefore, we investigated the functional role of TGIF-1 in placental angiogenesis and examined similar functions.

Microvascular ECs, which are found in the fetal capillaries of chorionic villi, exhibit a proliferative phenotype, as they play an important role in the vascularisation of the placenta (Thorin \& Shreeve 1998). TGIF-1 inactivation in HUVECs and SGHEC-7 cells significantly reduced their proliferative ability, while increasing the angiogenic potential of the ECs. This demonstrates that TGIF-1 plays a role in regulating the ability of ECs to form cell-cell and cell-matrix connections. The effect of TCIF-1 in vascular development is not surprising as mouse studies have found embryos lacking Tgif-1 to be extremely growth restricted with placental defects affecting the vasculature (Bartholin et al. 2008). The overexpression of TGIF-1 in human FGR placental endothelium suggests that there may be excessive proliferation with insufficient differentiation, resulting in impaired placental angiogenesis.

Another critical aspect of placental angiogenesis is the degradation of the basement membrane by proteases released by ECs (Kaufmann et al. 2004). Metalloproteinases (MMPs) are proteases that belong to a family of at least 15 secreted and membrane-bound zinc endopeptidases. The results of this study indicate a significant difference in MMP-2 activity in HUVECs and SGHEC-7 cells transfected with TGIF-1 siRNA compared with that in NC-transfected ECs. Other studies have found MMP-1, -2, -3, -9 and TIMP1 enzymatic activity to be associated with ECs (Hanemaaijer et al. 1993). However, under basal conditions without growth supplementation, MMP1 and MMP2 activity is evident but that of MMP9 is not, suggesting this is not constitutively secreted (Jackson \& Nguyen 1997). Therefore, it is possible that due to the use of serumfree medium in our experiments, no effect on MMP9 activity was observable. Dysfunctional secretion of these enzymes would significantly influence basement membrane degradation and invasive potential, which would affect the vasculature development.

To determine the role of TGIF- 1 as a functional regulator of angiogenesis, we investigated downstream targets of TGIF-1 in EC angiogenesis by utilising lowdensity angiogenesis-related PCR arrays to profile alterations in gene expression. The array consists of 92 angiogenesis-related genes targeting known angiogenic growth factors including VEGF, endostatin and cell adhesion molecules. In addition, the array contains markers and targets for angiogenesis and lymphangiogenesis. From the array, four candidate genes that showed altered expression after TCIF-1 inactivation were selected for further validation on independent cultures of TGIF-1-inactivated HUVECs and SGHEC-7 cells. NRP-1 and ITGAV showed increases in gene expression consistent with the array. ANGPT-1 mRNA expression was significantly decreased in HUVECs and SGHEC-7 cells, whereas the related ANGPT-2 showed an opposite increase in mRNA expression.

The TGIF-1 downstream target gene NRP-1 is an important regulator of angiogenesis particularly in the cardiovascular system (Kawakami et al. 2002). NRP-1 acts as a co-receptor for VEGF, which is a principal promoter of angiogenic processes and is involved in the differentiation, tube formation and vascular maturation of ECs (Flamme et al. 1997). From the low-density array, VEGF was also upregulated as a result of TGIF-1 silencing. Thus, it is speculated that NRP-1, in cooperation with VEGF, may help in regulating the formation of EC networks. Consequently, with overexpression of TGIF-1 in FGR placentae, the downstream NRP-1-targeted gene would be expected to be decreased. A recent study confirms this, demonstrating significantly reduced NRP-1 expression in placentae from human FGRaffected pregnancies with an absent end-diastolic flow in the umbilical artery (Maulik et al. 2016). Hence, TGIF-1 may be an important upstream regulator of placental angiogenesis.

Our study also identifies ITGAV as a downstream angiogenic target of TGIF-1. ITGAV codes for the $\alpha \mathrm{V}$ integrin and is involved in cell adhesion. Overexpression of TGIF-1 will lead to a reduction in ITGAV expression. Deletion of Itgav in mice shows impaired vascular development in the central nervous and the ophthalmic systems, similar to that seen in the deletion of Nrp-1 (Arnold et al. 2012). A major function of this integrin is to activate TGF $\beta 1$ signalling (Arnold et al. 2012), which is a crucial signalling pathway in placental development. Therefore, impaired placental vascularisation in human FGR may be a result of TGIF-1 overexpression reducing ITGAV expression.

The TGIF-1 downstream targets of ANGPT1 and ANGPT2 code for angiopoietins 1 and 2 respectively, and are critical mediators of vascular development. Angiopoietin 2 (ANGPT2) is an antagonist for both angiopoietin 1 (ANGPT1) and the TIE-2 receptor (Drenkhahn et al. 2004). ANGPT1 is known to provide a stabilising signal through the TIE-2 receptor, which can be blocked by ANGPT2 to prevent vascular sprouting only if VEGF is absent (Maisonpierre et al. 1997). Increased placental expression of TGIF-1 observed in human FGR is expected to upregulate ANGPT1 and downregulate ANGPT2 mRNA. Altered expression of both ANGPT1 and ANGPT2 is implicated in an ovine model of FGR (Hagen et al. 2005). Imbalances in the concentrations of angiopoietins 1 and 2 may contribute to the villous pathology of the FGR microvasculature via 
the induction of premature maturation of the terminal villi capillaries (Dunk et al. 2000). Thus, angiopoietin signalling may be another pathway through which pathological TGIF-1 overexpression impacts placental angiogenesis in human FGR.

In conclusion, this is the first study to report an increased expression of TGIF-1 in PLECs from FGRaffected pregnancies. In vitro functional analyses suggest that TGIF-1 regulates placental angiogenesis through effects on the ability of ECs to proliferate, form networks and invade. Increased expression of TGIF-1 in endothelial cells may contribute to reduced branching angiogenesis observed in FGR placentae. Our study also identified ITGAV, NRP-1, ANGPT1 and ANGPT2 as downstream targets of TGIF-1, which are important mediators of placental angiogenesis in FGR. Thus, the increased expression of homeobox gene TGIF-1 may be involved in the molecular mechanisms underlying the aberrant angiogenesis observed in human FGR.

\section{Declaration of interest}

The authors declare that there is no conflict of interest that could be perceived as prejudicing the impartiality of the research reported.

\section{Funding}

This work was supported by the Australian National Health and Medical Research Council (NHMRC project grant \#509140) to Dr P Murthi.

\section{Acknowledgements}

The authors would like to thank the patients and midwives who contributed to the placental sample collections.

\section{References}

Arnaoutova I \& Kleinman HK 2010 In vitro angiogenesis: endothelial cell tube formation on gelled basement membrane extract. Nature Protocols 5 628-635. (doi:10.1038/onc.2011.50)

Arnold TD, Ferrero GM, Qiu H, Phan IT, Akhurst RJ, Huang EJ \& Reichardt LF 2012 Defective retinal vascular endothelial cell development as a consequence of impaired integrin alphaVbeta8mediated activation of transforming growth factor-beta. Journal of Neuroscience 32 1197-1206. (doi:10.1210/en.2006-1248)

Bartholin L, Melhuish TA, Powers SE, Goddard-Leon S, Treilleux I, Sutherland AE \& Wotton D 2008 Maternal Tgif is required for vascularization of the embryonic placenta. Developmental Biology 319 285-297. (doi:10.1002/hep.22793)

Bouis D, Hospers GA, Meijer C, Molema G \& Mulder NH 2001 Endothelium in vitro: a review of human vascular endothelial cell lines for blood vessel-related research. Angiogenesis 4 91-102. (doi:10.1093/ toxsci/kfi044)

Dobbins TA, Sullivan EA, Roberts CL \& Simpson JM 2012 Australian national birthweight percentiles by sex and gestational age, 1998-2007. Medical Journal of Australia 197 291-294. (doi10.5694/mja11.11331)

Douville JM \& Wigle JT 2007 Regulation and function of homeodomain proteins in the embryonic and adult vascular systems. Canadian
Journal of Physiology and Pharmacology 85 55-65. (doi:10.1210/ en.2008-1518)

Drenkhahn M, Gescher DM, Wolber EM, Meyhoefer-Malik A \& Malik E 2004 Expression of angiopoietin 1 and 2 in ectopic endometrium on the chicken chorioallantoic membrane. Fertility and Sterility 81 869-875. (doi:10.1002/(ISSN)1097-0215)

Dunk C, Shams M, Nijjar S, Rhaman M, Qiu Y, Bussolati B \& Ahmed A 2000 Angiopoietin-1 and angiopoietin-2 activate trophoblast Tie-2 to promote growth and migration during placental development. American Journal of Pathology 156 2185-2199. (doi:10.1016/S1043-2760(98)00026-5)

Dunk CE, Roggensack AM, Cox B, Perkins JE, Åsenius F, Keating S, Weksberg R, Kingdom JC \& Adamson SL 2012 A distinct microvascular endothelial gene expression profile in severe IUGR placentas. Placenta 33 285-293. (doi:10.1016/j.placenta.2011.12.020)

Faresse N, Colland F, Ferrand N, Prunier C, Bourgeade MF \& Atfi A 2008 Identification of PCTA, a TGIF antagonist that promotes PML function in TGF-beta signalling. EMBO Journal 27 1804-1815. (doi:10.1158/00085472.CAN-12-2218)

Fickling SA, Tooze JA \& Whitley GS 1992 Characterization of human umbilical vein endothelial cell lines produced by transfection with the early region of SV40. Experimental Cell Research 201 517-521. (doi:10.1016/0014-4827(92)90303-p)

Fitzsimmons PJ, Forough R, Lawrence ME, Gantt DS, Rajab MH, Kim H, Weylie B, Spiekerman AM \& Dehmer GJ 2007 Urinary levels of matrix metalloproteinase 9 and 2 and tissue inhibitor of matrix metalloproteinase in patients with coronary artery disease. Atherosclerosis 194 196-203. (doi:10.1245/s10434-010-0956-9)

Flamme I, Frolich T \& Risau W 1997 Molecular mechanisms of vasculogenesis and embryonic angiogenesis. Journal of Cell Physiology 173 206-210. (doi:10.1002/(sici)1097-4652(199711)173:2<206::aidjcp22>3.0.co;2-c)

Gorski DH \& Walsh K 2000 The role of homeobox genes in vascular remodeling and angiogenesis. Circulation Research 87 865-872. (doi:10.1161/01.RES.87.10.865)

Hagen AS, Orbus RJ, Wilkening RB, Regnault TR \& Anthony RV 2005 Placental expression of angiopoietin-1, angiopoietin-2 and tie-2 during placental development in an ovine model of placental insufficiency-fetal growth restriction. Pediatric Research 58 1228-1232. (doi:10.1210/ jc.2007-0812)

Hamik A, Wang B \& Jain MK 2006 Transcriptional regulators of angiogenesis. Arteriosclerosis, Thrombosis, and Vascular Biology 26 1936-1947. (doi:10.4174/astr.2015.88.2.77)

Hanemaaijer R, Koolwijk P, le Clercq L, de Vree WJ \& van Hinsbergh VW 1993 Regulation of matrix metalloproteinase expression in human vein and microvascular endothelial cells. Effects of tumour necrosis factor alpha, interleukin 1 and phorbol ester. Biochemical Journal 296 803-809. (doi:10.1093/carcin/23.1.25)

Hayhurst M \& McConnell SK 2003 Mouse models of holoprosencephaly. Current Opinion in Neurology 16 135-141. (doi:10.1073/ pnas.230285997)

Holland PW, Booth HA \& Bruford EA 2007 Classification and nomenclature of all human homeobox genes. BMC Biology 5 47. (doi:10.1210/ en.2004-0612)

Jackson CJ \& Nguyen M 1997 Human microvascular endothelial cells differ from macrovascular endothelial cells in their expression of matrix metalloproteinases. International Journal of Biochemistry \& Cell Biology 29 1167-1177. (doi:10.1007/s12015-007-0006-6)

Kaufmann P, Mayhew TM \& Charnock-Jones DS 2004 Aspects of human fetoplacental vasculogenesis and angiogenesis. II. Changes during normal pregnancy. Placenta 25 114-126. (doi:10.1210/en.2012-1728)

Kawakami T, Tokunaga T, Hatanaka H, Kijima H, Yamazaki H, Abe Y, Osamura Y, Inoue H, Ueyama Y \& Nakamura M 2002 Neuropilin 1 and neuropilin 2 co-expression is significantly correlated with increased vascularity and poor prognosis in nonsmall cell lung carcinoma. Cancer 95 2196-2201. (doi:10.1002/cncr.10936)

Kingdom J, Huppertz B, Seaward G \& Kaufmann P 2000 Development of the placental villous tree and its consequences for fetal growth. European Journal of Obstetrics, Gynecology, and Reproductive Biology 92 35-43. (doi:10.1007/s10552-007-9024-6)

Latchman DS 1997 Transcription factors: an overview. International Journal of Biochemistry \& Cell Biology 29 1305-1312. (doi:10.1016/ j.jhep.2010.04.028) 
Lepparanta O, Pulkkinen V, Koli K, Vahatalo R, Salmenkivi K, Kinnula VL, Heikinheimo M \& Myllarniemi M 2010 Transcription factor GATA6 is expressed in quiescent myofibroblasts in idiopathic pulmonary fibrosis. American Journal of Respiratory Cell and Molecular Biology 42 626-632. (doi:10.1074/jbc.M806548200)

Livak KJ \& Schmittgen TD 2001 Analysis of relative gene expression data using real-time quantitative PCR and the 2(-Delta Delta $\mathrm{C}(\mathrm{T})$ ) method. Methods 25 402-408. (doi:10.1016/j.mce.2009.08.028)

Maisonpierre PC, Suri C, Jones PF, Bartunkova S, Wiegand SJ, Radziejewski C, Compton D, McClain J, Aldrich TH, Papadopoulos N et al. 1997 Angiopoietin-2, a natural antagonist for Tie2 that disrupts in vivo angiogenesis. Science 277 55-60. (doi:10.1053/ j.gastro.2010.01.041)

Maulik D, De A, Ragolia L, Evans J, Grigoryev D, Lankachandra K, Mundy D, Muscat J, Gerkovich MM \& Ye SQ 2016 Downregulation of placental neuropilin-1 in fetal growth restriction. American Journal of Obstetrics and Gynecology 214 e1-e9. (doi:10.1007/s00418-0080482-z)

Murthi P, Doherty V, Said J, Donath S, Brennecke SP \& Kalionis B 2006 Homeobox gene HLX1 expression is decreased in idiopathic human fetal growth restriction. American Journal of Pathology 168 511-518. (doi:10.2353/ajpath.2006.050637)

Murthi P, Hiden U, Rajaraman G, Liu H, Borg AJ, Coombes F, Desoye G, Brennecke SP \& Kalionis B 2008 Novel homeobox genes are differentially expressed in placental microvascular endothelial cells compared with macrovascular cells. Placenta 29 624-630. (doi:10.1172/JCI19479)

Murthi P, So M, Gude NM, Doherty VL, Brennecke SP \& Kalionis B 2007 Homeobox genes are differentially expressed in macrovascular human umbilical vein endothelial cells and microvascular placental endothelial cells. Placenta 28 219-223.

Nakagawa T, Abe M, Yamazaki T, Miyashita H, Niwa H, Kokubun S \& Sato Y 2003 HEX acts as a negative regulator of angiogenesis by modulating the expression of angiogenesis-related gene in endothelial cells in vitro. Arteriosclerosis, Thrombosis, and Vascular Biology 23 231-237. (doi:10.1161/01.atv.0000052670.55321.87)
Pathirage NA, Cocquebert M, Sadovsky Y, Abumaree M, Manuelpillai U, Borg A, Keogh RJ, Brennecke SP, Evain-Brion D, Fournier T et al. 2013 Homeobox gene transforming growth factor beta-induced factor-1 (TGIF-1) is a regulator of villous trophoblast differentiation and its expression is increased in human idiopathic fetal growth restriction. Molecular Human Reproduction 19 665-675. (doi:10.1016/S00225347(05)64161-4)

Rajaraman G, Murthi P, Pathirage N, Brennecke SP \& Kalionis B 2010 Downstream targets of homeobox gene HLX show altered expression in human idiopathic fetal growth restriction. American Journal of Pathology 176 278-287. (doi:10.2353/ajpath.2010.090187)

Reynolds LP \& Redmer DA 2001 Angiogenesis in the placenta. Biology of Reproduction 64 1033-1040. (doi:10.1002/hep.24740)

Rossant J \& Cross JC 2001 Placental development: lessons from mouse mutants. Nature Reviews. Genetics 2 538-548. (doi:10.1038/35080570)

Su EJ, Xin H, Yin P, Dyson M, Coon J, Farrow KN, Mestan KK \& Ernst LM 2015 Impaired fetoplacental angiogenesis in growth-restricted fetuses with abnormal umbilical artery doppler velocimetry is mediated by aryl hydrocarbon receptor nuclear translocator (ARNT). Journal of Clinical Endocrinology and Metabolism 100 E30-E40. (doi:10.1002/ (ISSN)1098-2744)

Thorin E \& Shreeve SM 1998 Heterogeneity of vascular endothelial cells in normal and disease states. Pharmacology Therapy 78 155-166. (doi:10.1038/onc.2013.309)

Yaron Y, McAdara JK, Lynch M, Hughes E \& Gasson JC 2001 Identification of novel functional regions important for the activity of HOXB7 in mammalian cells. Journal of Immunology $\mathbf{1 6 6} \quad 5058-5067$. (doi:10.1016/j.cancergencyto.2009.09.010)

Received 17 February 2016

First decision 7 March 2016

Revised manuscript received 18 July 2016

Accepted 18 August 2016 